\title{
Prospective Evaluation of Neuromediator Dynamics in Castration-Resistant Prostate Cancer Patients During Docetaxel
}

\author{
JOST VON HARDENBERG ${ }^{1}$, MAIKE SCHWARTZ ${ }^{1}$, THORSTEN WERNER ${ }^{2}$, \\ STEFAN FUXIUS ${ }^{3}$, MARKUS MÜLLER ${ }^{4}$, THOMAS FRANGENHEIM ${ }^{5}$, \\ CHRISTIAN BOLENZ $^{1 *}$, CHRISTEL WEISS ${ }^{6}$ and ELMAR HEINRICH ${ }^{7 \#}$ \\ ${ }^{1}$ Department of Urology, University Medical Center Mannheim, University of Heidelberg, Mannheim, Germany; \\ ${ }^{2}$ Outpatient Urology Practice, Herzberg am Harz, Germany; \\ ${ }^{3}$ Outpatient Oncology Practice Heidelberg, Heidelberg, Germany; \\ ${ }^{4}$ Department of Urology, Hospital Ludwigshafen, Ludwigshafen, Germany; \\ ${ }^{5}$ Outpatient Urology Practice, Bruchsal, Germany; \\ ${ }^{6}$ Department of Medical Statistics and Biomathematics, Medical Faculty \\ Mannheim, University of Heidelberg, Mannheim, Germany; \\ ${ }^{7}$ Department of Urology, University Hospital Goettingen, Goettingen, Germany
}

\begin{abstract}
Aim: Aim of the study was to detect small cell/neuroendocrine (SCNC) transformation in metastatic castration-resistant prostate cancer $(m C R P C)$ that is a challenging procedure. We investigated the role of neuromediator dynamics as potential evidence of SCNC in patients undergoing docetaxel therapy. Patients and Methods: A multi-institutional, prospective observational study was conducted. Patients undergoing docetaxel treatment were included. Chromogranin A (CGA), neuron-specific enolase (NSE), and pro-gastrin releasing peptide (Pro-GRP) were sequentially evaluated at predefined time points. Outcome measures were overall survival (OS), progression-free survival (PFS) and PSA nadir. Results: Fifty-two patients were included. A general rise in CGA levels was observed. Patients with a high CGA rise (100\%ULN: CGA $\geq 98.1 \mathrm{ng} / \mathrm{ml})$ between
\end{abstract}

*Current address: Department of Urology, University Hospital Ulm, Ulm, Germany.

\#Current address: Department of Urology, Hospital Wels-Grieskirchen, Wels, Austria.

Correspondence to: Elmar Heinrich, MD, Department of Urology, Hospital Wels-Grieskirchen, Grieskirchner Str. 42, 4600 Wels, Austria. Tel: +43 72424152328, Fax: +43 72424153774, e-mail: elmar.heinrich@gmx.de

Key Words: Castration-resistant prostate cancer, docetaxel, neuroendocrine transformation, chromogranin A, neuron-specific enolase, pro-gastrin-releasing peptide. the 1st and 3rd cycle trended towards a decreased OS $(p=0.0649)$ and showed a decreased PFS ( $p=0.0369)$. In multivariate analysis, continuous CGA rise correlated with PFS ( $p=0.0553$; HR 1.136), but was not an independent predictor of OS. Conclusion: Patients with an early high CGA rise may demonstrate a subgroup with poor outcome due to underlying SCNC transformation. Monitoring of CGA appears to be an option worth considering.

The wide range of survival of patients with $\mathrm{mCRPC}$ is, at least partly, attributed to tumor heterogeneity. Its identification, prognostic impact, and consequent influence in treatment remain a leading challenge in mCRPC.

The development of CRPC is caused by androgendeprivation therapy (ADT) (1). Even though the first therapeutic hit with ADT is highly efficient, therapy resistance develops. This generates molecular changes of the androgen receptor axis (ARA) (1). An interference with the ARA also increases the emergence of SCNC during ADT (24). ARA-targeted agents abiraterone acetate (AA) and enzalutamide (ENZ) were granted approval. An ongoing prospective multicenter trial is evaluating the emergence of SCNC by biopsies in MCRPC that have progressed under treatment with AA or ENZ. First results show a SCNC or an intermediate atypical carcinoma in $40 \%$ of samples being significantly correlated with a decreased overall survival and the elevation of CGA and NSE (5). It has been shown that already CGA-positive cell clusters represent an early sign of invasion (6). The prognosis of SCNC is poor due to late recognition and heterogeneous clinical features (7). 
The microtubule-stabilizing, semi-synthetic taxane DOC is the standard first-line chemotherapy for $\operatorname{CRPC}(8,9)$. Earlier, researchers suggested that treatment with DOC significantly impairs the ARA by interfering with AR nuclear transport in CRPC $(10,11)$. In vitro findings showed that the acquisition of a neuroendocrine phenotype by prostate cancer cells leads to resistance of DOC (12).

Neuroendocrine cells synthesize several neuromediators. To detect neuroendocrine areas in prostate cancer, the most recent methodological directive suggests that any evaluation of the three common markers (CGA, CD56 or SYN) is sufficient. Although the diagnosis of SCNC is a priori a pathological diagnosis (13), there is good evidence in support of a significant relationship between neuromediator levels, tissue expression, and neuroendocrine activity in prostate cancer patients $(5,14,15)$.

With growing emergence of SCNC, and possible treatment resistance to conventional therapies, it is important to identify patient cohorts that might benefit from biopsy sampling of metastases to detect SCNC transformation. Early detection of SCNC would result in change of therapy.

Here, we hypothesized that pretreatment or treatment with DOC may have influenced neuromediators, which, in turn, may impact patient outcome. To test our hypothesis, we conducted a prospective, multi-institutional observational study to sequentially evaluate serum neuromediators during DOC in mCRPC.

\section{Patients and Methods}

Study design and patients. Our investigator-initiated study was designed and coordinated by $\mathrm{JvH}, \mathrm{EH}$ and $\mathrm{CW}$. We conducted a prospective, observational ethics-committee-approved study at six institutions (two tertiary centers, one teaching hospital, and three outpatient practices) with the primary objective of evaluating dynamics from baseline of neuromediators in serum of patients with mCRPC undergoing DOC chemotherapy. Secondary objectives included to detect correlations of changes in neuromediators for OS, PFS and PSA nadir. The study is registered with Germanctr.de, number DRKS00004797.

Eligibility criteria were histologically-confirmed adenocarcinoma, mCRPC (newly diagnosed or with progression after regimens with $\mathrm{AA}$ and/or ENZ and/or DOC). Exclusion criteria were any history of cancer apart from low grade urothelial cancer of the bladder within the last 5 years; neuroendocrine cancer in the past medical history. Potential clinical manifestations associated with SCNC were scored as described previously (13). All study participants provided written informed consent after full explanation of the purpose and nature of all procedures.

Patients received DOC $75 \mathrm{mg} / \mathrm{m}^{2}$ intravenously 3-weekly. The dose of DOC was reduced if toxic effects deemed unacceptable were noted. The decision to end treatment due to toxic effects, progression, or other reason was in hands of the treating physician.

Serum collection, measurement and storage. Blood was collected in serum-gel tubes during routine blood withdrawal within seven days

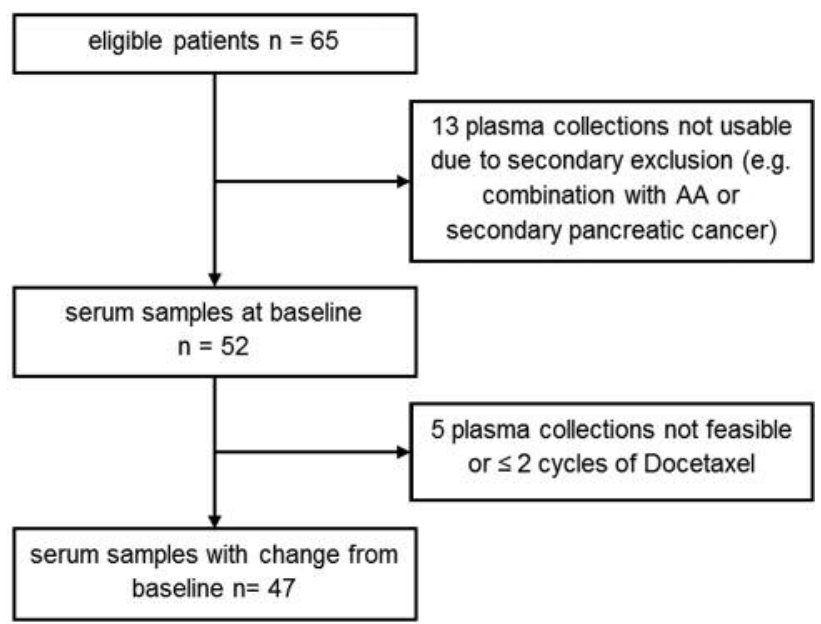

Figure 1. CONSORT diagram on enrollment and attrition.

before application of the $1 \mathrm{st}$, the $3 \mathrm{rd}$, and the 6 th cycle of systemic chemotherapy with DOC. In case of dose modifications, the blood withdrawal (bw) scheme was adapted. All serum samples were sent by medical express service to a reference laboratory. For quantitative determination of CGA the Time-Resolved Amplified Cryptate Emission (TRACE) method (Cisbio Bioassays, France), of NSE the chemoluminescence immunoassay (CLIA) LIAISON ${ }^{\circledR}$ NSE (DiaSorin, Italy) and of Pro-GRP the chemiluminescent microparticle immunoassay (CMIA) (Abbot, Germany) were used.

Statistical analysis. First, we calculated the change from baseline of neuromediators under DOC. From clinical experience and reported case series, we assumed that at least a total increase of $100 \%$ of the upper limit of normal (ULN) of the widely used serum markers CGA and NSE would be clinically relevant (high rise). Second, we therefore investigated the correlation of an early high rise and an early change (1st - 3rd cycle) from baseline for OS, PFS and PSA nadir. For OS, patients were followed until the date of death from any cause. Patients who did not experience the event by the end of the study were censored at the time of last available follow-up. We analyzed PFS, and PSA nadir according to recent recommendations (16).

All statistical calculations were conducted on $\mathrm{SAS}^{\circledR}$ release 9.3 (SAS Institute Inc., NC, USA). For categorical factors, absolute frequencies and relative proportions are given; approximately normally distributed variables (i.e. age) are presented by mean value and standard deviation; for skewed variables, median and range are given. In order to compare measurements over time, repeated measures ANOVA was performed using the SAS procedure PROC MIXED. If the ANOVA yielded a statistically significant result Dunnett's test was used as a post hoc test for pairwise comparisons. The test was conducted as two-sided tests. For these techniques, the logarithm of marker values were used in order to get approximately normally distributed data.

Furthermore, Kaplan-Meier curves were created to illustrate event rates. The Gehan-Wilcoxon test was used to investigate the influence of an early high rise of CGA and NSE for OS and PFS. As the beginning of the observation is crucial for the patient's course of the 
Table I. Characterization of the study cohort.

\begin{tabular}{|c|c|}
\hline Patient characteristics $(\mathrm{N}=52)$ & $\mathrm{N}(\%)$ \\
\hline \multicolumn{2}{|l|}{ Age (years) } \\
\hline Mean \pm SD & $71.3 \pm 7.2(55-88)$ \\
\hline \multicolumn{2}{|l|}{ ECOG performance status } \\
\hline 0 & $34(65.4 \%)$ \\
\hline 1 & $19(34.6 \%)$ \\
\hline \multicolumn{2}{|l|}{ Charlson comorbidity index } \\
\hline$<2$ & $42(90.8 \%)$ \\
\hline$\geq 2$ & $10(19.2 \%)$ \\
\hline \multicolumn{2}{|l|}{$\begin{array}{l}\text { First diagnosis prostate cancer - } \\
\text { study entry (months) }\end{array}$} \\
\hline median (range) & $34(6-220)$ \\
\hline \multicolumn{2}{|l|}{$\begin{array}{l}\text { Gleason score in biopsy/ } \\
\text { surgical specimen (missing } n=5 \text { ) }\end{array}$} \\
\hline 6 & $2(4.3 \%)$ \\
\hline 7 & $12(26.7 \%)$ \\
\hline 8 & $12(26.7 \%)$ \\
\hline 9 & $20(42.6 \%)$ \\
\hline 10 & $1(2.2 \%)$ \\
\hline \multicolumn{2}{|l|}{ Site of metastatic disease* } \\
\hline Bone & $42(80.8 \%)$ \\
\hline Visceral metastasis & $14(26.9 \%)$ \\
\hline Lymph node metastasis & $35(67.3 \%)$ \\
\hline Clinical manifestation of NED & $45(86.5 \%)$ \\
\hline \multicolumn{2}{|l|}{ Prior systemic treatment* } \\
\hline Androgen deprivation therapy & $52(100 \%)$ \\
\hline Months, median (range) & $24(3-139)$ \\
\hline Docetaxel & $4(7.7 \%)$ \\
\hline Abiraterone Acetate & $24(46.2 \%)$ \\
\hline Enzalutamide & $7(13.5 \%)$ \\
\hline \multicolumn{2}{|l|}{ Blood based marker } \\
\hline Hemoglobin (Hb), g/dl & $12.1(7.0-15.1)$ \\
\hline Alkaline phosphatase (AP), U/I & $139(46-2,226)$ \\
\hline Lactate dehydrogenase (LDH), U/I & $276(128-1,586)$ \\
\hline Intake of proton pump inhibitors & $16(30.8 \%)$ \\
\hline
\end{tabular}

Data are number (\%) unless stated otherwise. ECOG=Eastern Cooperative Oncology Group; *patients could have more than one.

disease, the Gehan-Wilcoxon test seems to be appropriate because it gives greater weight to the earlier differences at the beginning of the observation than the log rank or the cox regression analysis (17). Influences for OS and PFS were evaluated by Cox regression models for hazard ratios (HR) and 95\% confidence interval (CI). All variables in the univariate analysis with a significant test result were included in the multivariable model (using the "selection=forward" method with alpha $=0.10$ ). To test for influence of the variables on PSA nadir, the Mann-Whitney $U$-test was used.

A test result was considered statistically significant for $p<0.05$. A test result with $0.05<p<0.10$ has been regarded as trending towards significance.

\section{Results}

Study population. Between February 2013 and July 2015, 65 patients met the eligibility criteria and were included in the
Table II. Marker distribution (bw: blood withdrawal).

\begin{tabular}{|c|c|c|c|}
\hline \multirow[t]{2}{*}{ Marker } & $1 \mathrm{st} b w$ & 2nd bw & $3 \mathrm{rd} \mathrm{bw}$ \\
\hline & \multicolumn{3}{|c|}{ Median (range) } \\
\hline CGA (ng/ml) & $\begin{array}{c}132(22-1023) \\
(\mathrm{n}=49)\end{array}$ & $\begin{array}{c}146(12-2027) \\
(\mathrm{n}=48)\end{array}$ & $\begin{array}{c}160(39-961) \\
(\mathrm{n}=39)\end{array}$ \\
\hline NSE (ng/ml) & $\begin{array}{c}17(10-60) \\
(\mathrm{n}=49)\end{array}$ & $\begin{array}{c}19(6-117) \\
(\mathrm{n}=45)\end{array}$ & $\begin{array}{c}15(9-68) \\
(\mathrm{n}=37)\end{array}$ \\
\hline Pro-GRP $(\mu \mathrm{g} / \mathrm{ml})$ & $\begin{array}{c}19(6-122) \\
(\mathrm{n}=49)\end{array}$ & $\begin{array}{c}15(3-77) \\
(\mathrm{n}=47)\end{array}$ & $\begin{array}{c}15(5-36) \\
(\mathrm{n}=36)\end{array}$ \\
\hline PSA (ng/ml) & $\begin{array}{c}143(0-2525) \\
(\mathrm{n}=52)\end{array}$ & $\begin{array}{c}62.4(0-2164) \\
(\mathrm{n}=46)\end{array}$ & $\begin{array}{c}22(0-1765) \\
(\mathrm{n}=37)\end{array}$ \\
\hline
\end{tabular}

study. Thirteen patients had to be excluded secondarily due to e.g. combination with AA or primary hormone chemotherapy (Figure 1). The clinical and pathological data of all patients are listed in Table I. Thirty-seven $(71.1 \%)$ patients had died at the time of analysis. For $19(47.5 \%)$, PFS could be provided, whereas 21 (52.5\%) patients were censored.

Expression and dynamics of neuromediators. Serum concentrations of CGA, NSE, Pro-GRP, and PSA at all time points of bw are given in Table II. The distribution of CGA, NSE and Pro-GRP is shown in boxplots in Figure 2. Levels of CGA increased significantly from baseline compared to the 2 nd and the 3rd blood withdrawal $(p=0.0146$ and $p=0.0330)$. Levels of NSE did not change during therapy with DOC $(p=n . s$.$) . Levels of Pro-GRP$ significantly decreased during therapy compared to baseline $(p=0.0424$ and $p=0.0114)$. Waterfall plots depicting changes of the markers are presented in Figure 3. A total increase $\geq 100 \%$ of the upper limit of normal (ULN) of CGA was observed in 11 patients and of NSE in 5 patients. The total change was shown for Pro-GRP, as no reference range exists.

Associations of neuromediators dynamics with clinical outcome. A high rise from baseline of CGA trended towards significance with OS ( $p=0.0649)$. A high change of NSE was not associated with OS $(p=0.7744)$. A high rise from baseline of CGA was associated with PFS $(p=0.0369)$. A high change of NSE not associated with PFS $(p=0.2895)$. Kaplan-Meier curves for association of early high rise of CGA with OS and PFS are shown in Figure 4. Cox regression analysis for associations of change of neuromediators and other variables with OS and PFS are presented in Table III and Table IV. PSA-nadir was associated with previous therapy with AA $(p=0.0285), \mathrm{ENZ}$ $(p=0.0593)$, and AP-levels at baseline (0.0386). 

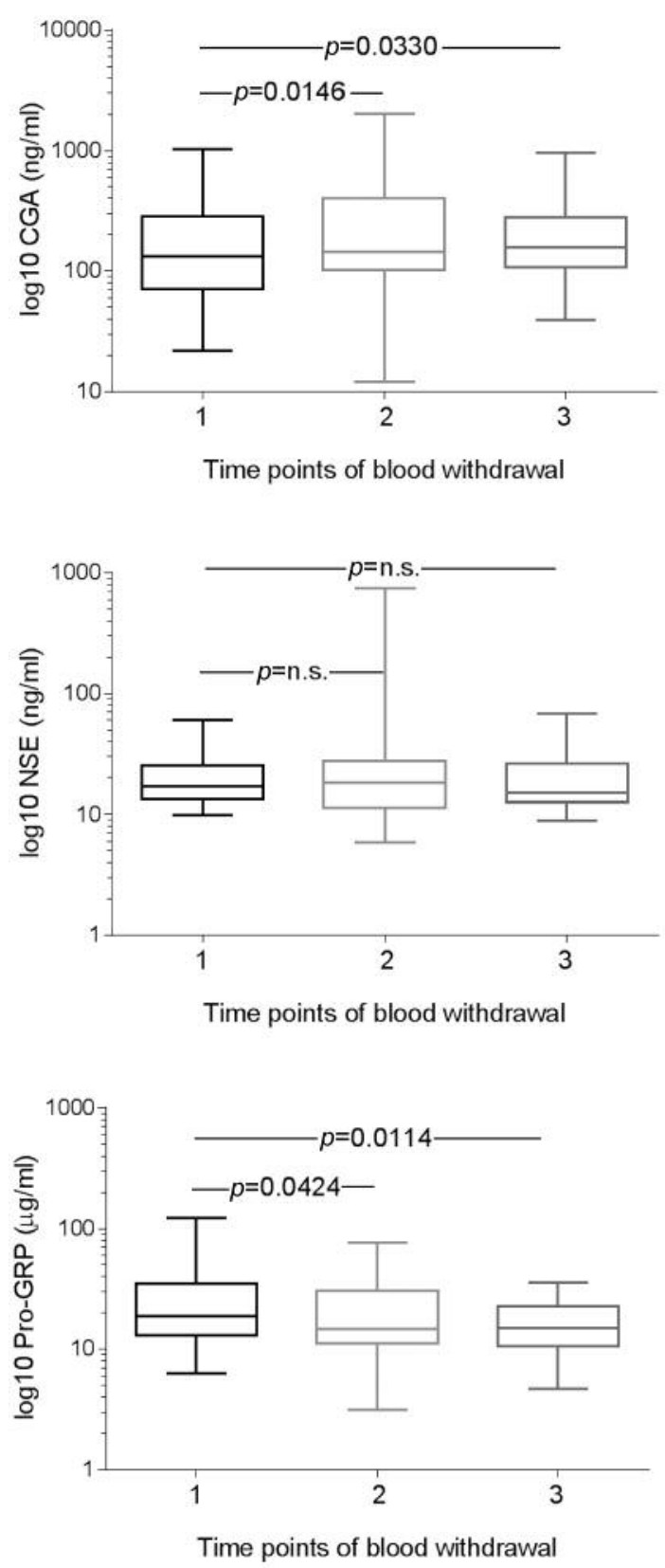

Figure 2. Boxplots for change of CGA, NSE, and Pro-GRP during therapy with $D O C$.

\section{Discussion}

We present here the first prospective study evaluating dynamics in serum neuromediators in men with mCRPC undergoing DOC. We found that CGA levels rise during therapy with DOC, but only an early high rise in CGA levels

trended towards significance with shorter OS and was associated with PFS indicating potential underlying SCNC transformation in a small subgroup. We observed that dynamics of NSE vary, whereas those of Pro-GRP decrease.

Figure 3. Waterfall diagrams for change from baseline during therapy with DOC (1st to 3rd cycle): (A) CGA with dashed line signifies total rise of $\geq 98.1 \mathrm{ng} / \mathrm{ml},(B) \mathrm{NSE}$ with dashed line signifies total rise of $\geq 18.3 \mathrm{ng} / \mathrm{ml}$, (C) Pro-GRP. 
Table III. Cox regression analysis for associations of change (1st to 3rd cycle) of CGA, NSE, Pro-GRP, and further clinical and laboratory variables with $O S$.

\begin{tabular}{|c|c|c|c|c|c|c|c|}
\hline \multirow[t]{2}{*}{ Variable } & \multirow[t]{2}{*}{ Categories } & \multicolumn{3}{|c|}{ Univariate analysis } & \multicolumn{3}{|c|}{ Multivariate analysis } \\
\hline & & $\mathrm{N}$ & HR $(95 \%$ CI $)$ & $p$-Value & $\mathrm{N}$ & $\operatorname{HR}(95 \% \mathrm{CI})$ & $p$-Value \\
\hline CGA change; 100 units & Continuous & 43 & $1.069(0.926-1.235)$ & 0.3633 & & & \\
\hline NSE change; 20 units & Continuous & 42 & $0.956(0.827-1.105)$ & 0.5419 & & & \\
\hline Pro-GRP change; 10 units & Continuous & 43 & $0.986(0.764-1.272)$ & 0.9110 & & & \\
\hline PSA; 100 units & Continuous & 50 & $1.075(1.019-1.133)$ & 0.0076 & 46 & $1.085(1.020-1.153)$ & 0.0155 \\
\hline AP; 100 units & Continuous & 48 & $1.121(1.046-1.200)$ & 0.0011 & 46 & $1.136(1.020-1.153)$ & 0.0002 \\
\hline $\mathrm{Hb}$ & Continuous & 51 & $0.830(0.695-0.992)$ & 0.0403 & & & \\
\hline LDH; 100 units & Continuous & 46 & $1.117(1.005-1.242)$ & 0.0402 & & & \\
\hline Age & Continuous & 52 & $1.047(0.994-1.104)$ & 0.0856 & 46 & $1.052(0.997-1.109)$ & 0.0609 \\
\hline ECOG & Binary 0 vs. $\geq 1$ & 34 vs. 19 & $1.322(0.660-2.645)$ & 0.4310 & & & \\
\hline AA therapy & Binary & & $1.805(0.921-3.536)$ & 0.0855 & & & \\
\hline
\end{tabular}

Table IV. Cox regression analysis for associations of change (1st to 3rd cycle) of CGA, NSE, Pro-GRP, and further clinical and laboratory variables with PFS.

\begin{tabular}{|c|c|c|c|c|c|c|c|}
\hline \multirow[t]{2}{*}{ Variable } & \multirow[t]{2}{*}{ Categories } & \multicolumn{3}{|c|}{ Univariate analysis } & \multicolumn{3}{|c|}{ Multivariate analysis } \\
\hline & & $\mathrm{N}$ & $\operatorname{HR}(95 \% \mathrm{CI})$ & $p$-Value & $\mathrm{N}$ & $\mathrm{HR}(95 \% \mathrm{CI})$ & $p$-Value \\
\hline CGA change; 100 units & Continuous & 37 & $1.136(0.999-1.291)$ & 0.0520 & 34 & $1.136(0.999-1.291)$ & 0.0520 \\
\hline NSE change; 20 units & Continuous & 33 & $0.960(0.804-1.146)$ & 0.6516 & & & \\
\hline Pro-GRP change; 10 units & Continuous & 34 & $1.121(0.736-1.708$ & 0.5946 & & & \\
\hline PSA; 100 units & Continuous & 39 & $1.031(0.956-1.112)$ & 0.4303 & & & \\
\hline AP; 100 units & Continuous & 39 & $1.042(0.895-1.214)$ & 0.5952 & & & \\
\hline $\mathrm{Hb}$ & Continuous & 39 & $0.806(0.606-1.072)$ & 0.1384 & & & \\
\hline LDH; 100 units & Continuous & 36 & $1.070(0.920-1.245)$ & 0.3782 & & & \\
\hline Age & Continuous & 39 & $1.013(0.943-1-089)$ & 0.7174 & & & \\
\hline ECOG & Binary $0 v s . \geq 1$ & 39 & $2.027(0.727-5.652)$ & 0.1767 & & & \\
\hline AA therapy & Binary & 39 & $1.673(0.632-4.428)$ & 0.3003 & & & \\
\hline
\end{tabular}

Aparicio et al. suggested potential clinical manifestations associated with SCNC (18). We applied these criteria and discovered that a majority of patients $(86.5 \%)$ have these features. The role of DOC as a putative driver of transformation to SCNC was not studied, although about one-third of DOC-pretreated men were found to have increased neuroendocrine marker levels (19). Based on our analysis DOC drives CGA levels, generally, during the course of therapy. During DOC-rechallenge combined with carboplatin, 33 patients were followed for CGA and NSE change, but the change was not correlated with time to progression or survival (20). In a small pilot study investigating Octreotide acetate in CRPC, CGA decrease during therapy could not identify clinical responders, but the study is biased by the small sample size $(n=17)(21)$. In gastroenteropancreatic neuroendocrine tumors change of CGA predicted treatment response (22). As the general increase of CGA in our study was not correlated to disease outcome, we believe that it may show a neuroendocrine stress response of neuroendocrine cells within the tumor or in general, but it should not be interpreted that DOC is a general driver of SCNC transformation.

Few patients showed a high rise of CGA during DOC. SCNC transformation in mCRPC is a histological diagnosis (13). To date, circulating tumor cells as an alternative are still highly experimental biomarkers and not used in the clinic routinely (23). Therefore, to identify SCNC transformation, sequential biopsies of metastases would be needed prior to several lines of therapy. Single core biopsy sampling may not be an accurate representation of the tumor as a whole due to intratumor heterogeneity shown in advanced prostate cancer (24). Small et al. reported that both visceral metastases and lymph node metastases can harvest SCNC (5). Due to the invasive and sometime cost-intensive (if CT-guided) procedure, 

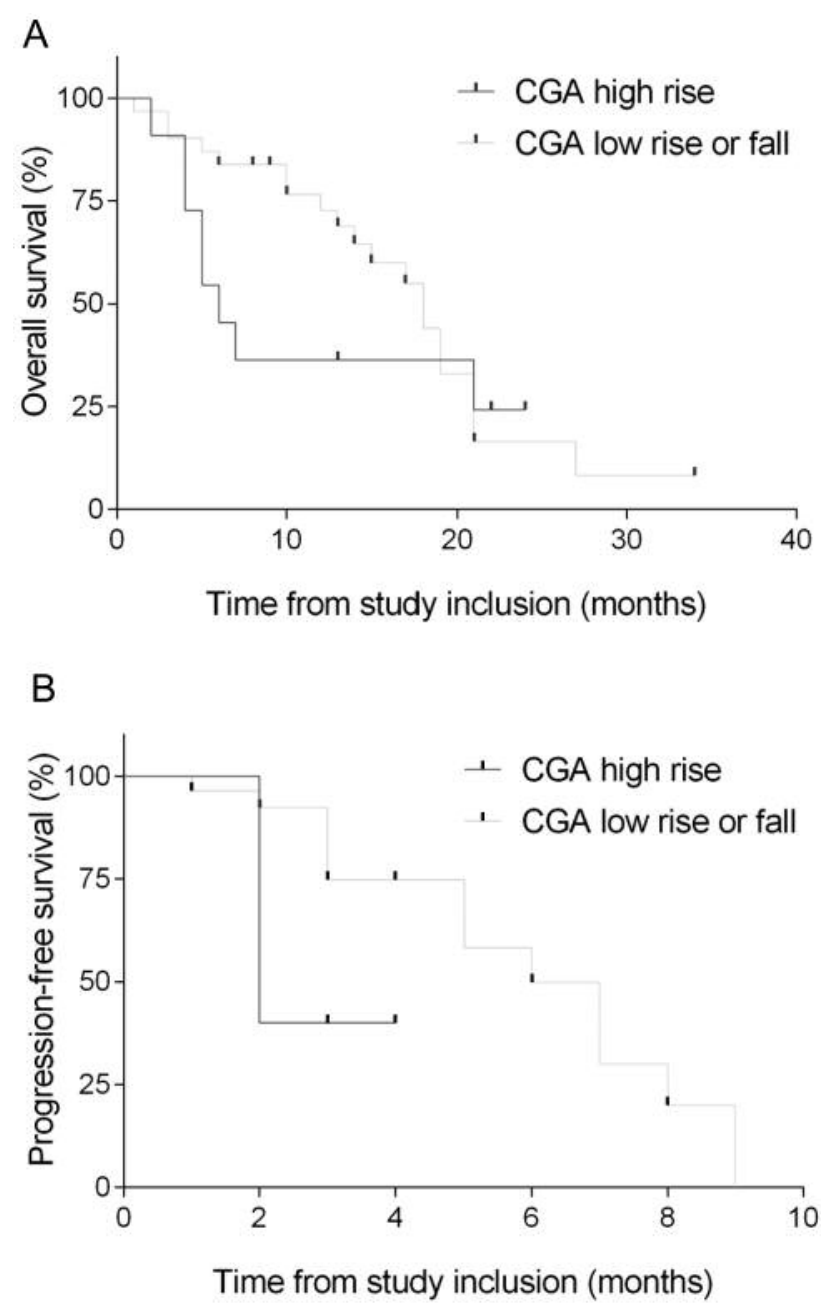

Figure 4. Kaplan-Meier curves for the association of CGA with OS (A) and $\operatorname{PFS}(B)$.

a preselection of patients at risk for SCNC transformation using circulating neuromediators could be useful.

From the immunohistochemical markers listed above, only CGA can be used as an established serum biomarker. Eleven patients experienced an early high rise of CGA, which was correlated with PFS and OS. Circulating CGA was used as objective tumor biology assessment tool in five trials including $>500$ patients with mCRPC in total according to a recent review (25). A high rise has not been studied in these trials. In the literature, a predominance of arbitrary thresholds were chosen after one-time analysis before therapy. Two recently published trials analyzed CGA only before AA therapy and found predictive and prognostic significance $(26,27)$. In our study some patients in the subgroup of patients with early high CGA rise still lived long. This could be in line with a review of Wang et al. that analyzed the clinical course of patients after diagnosis of neuroendocrine prostate cancer (7). Approximately $20 \%$ of the patients lived longer than 12 months, making the statistical analysis with our sample size for OS difficult. In the multivariate analysis, markers for the general volume of the disease were prognostic and CGA failed to show independent significance. Dynamics might have fewer biases as they are compared to baseline levels. Second dynamics can reflect the activity of the compartment under therapy. An early high rise of CGA should be further tested as a preselection tool for multi-core biopsy-sampling of metastases.

NSE and Pro-GRP are not recommended in immunohistochemistry, but they are serum-based and provide information about dynamics of other SCNC cell types. We were unable to detect any relevant associations of NSE with disease outcome. NSE in comparison to CGA showed to be a superior marker in small cell neuroendocrine cancers, whereas CGA was more sensitive in large cell neuroendocrine cancers (28). An explanation could, therefore, be that NSE is expressed by an aggressive small cell subtype of neuroendocrine cells and develops late in CRPC. The GRP/Bombesin receptor was shown to be over expressed in malignant prostate cells, and secretion of GRP by neuroendocrine cells in a paracrine manner might be one escape mechanism for prostate cancer when in an androgen independent stage (29). The growing field of GRP/Bombesin-based imaging for prostate cancer raises the need for more detailed knowledge concerning the role of ProGRP/GRP in the development and progression of prostate cancer (30). Yashi et al. found similarly to our results, increased Pro-GRP values in CRPC patient and observed also a decrease of Pro-GRP during ADT (31).

This analysis has limitations. No tandem evaluation of neuroendocrine serum markers and biopsy immunostaining in parallel was performed. Tandem evaluation was suggested to be the most reliable method to assess transformation to SCNC in mCRPC (15). Secondly, the patients did not receive a standardized staging protocol. Therefore radiographic progression-free survival could not be assessed. Thirdly, due to the small sample size it was difficult to gain significant results. Further larger studies are required to confirm our results.

Our prospective study demonstrated that dynamics of neuromediators vary, but early high CGA rise independently from PSA may indicate patients with poor outcome. Through the established low-cost serum marker CGA, the early reanalysis to assess dynamics could easily be used in daily practice. The high rise is possibly due to expression of a subclone of prostate cancer cells with SCNC transformation. Therefore, the cohort might benefit from multi-core biopsy sampling to detect SCNC transformation, if our results have been validated in larger cohorts. The ability to detect and monitor tumor heterogeneity like SCNC transformation remains a leading challenge for patients suffering from mCRPC. 


\section{Conflicts of Interest}

$\mathrm{EH}$ and $\mathrm{JvH}$ have received speaker honoraria from Sanofi-Aventis. All remaining authors have declared no conflicts of interest.

\section{Funding}

This work was supported by Sanofi-Aventis Deutschland GmbH. The study sponsor had no role in the study design, data collection, data analysis, data interpretation, or writing of the report.

\section{Acknowledgements}

The Authors thank the patients who volunteered to participate in this study. We would also like to acknowledge Annette Steidler, Karin Berner-Leischner, Dr. Petra Unglaub, Stanka Nikolic and Mrs. Löschner for supporting the course of the study.

\section{References}

1 Wyatt AW and Gleave ME: Targeting the adaptive molecular landscape of castration-resistant prostate cancer. EMBO Mol Med 7: 878-894, 2015.

2 Ismail A HR, Landry F, Aprikian AG and Chevalier S: Androgen ablation promotes neuroendocrine cell differentiation in dog and human prostate. Prostate 51: 117-125, 2002.

3 Ito T, Yamamoto S, Ohno Y, Namiki K, Aizawa T, Akiyama A and Tachibana M: Up-regulation of neuroendocrine differentiation in prostate cancer after androgen deprivation therapy, degree and androgen independence. Oncol Rep 8: 1221-1224, 2001.

4 Sasaki T, Komiya A, Suzuki H, Shimbo M, Ueda T, Akakura K and Ichikawa T: Changes in chromogranin a serum levels during endocrine therapy in metastatic prostate cancer patients. Eur Urol 48: 224-230, 2005.

5 Small EJ, Huang J, Youngren J, Sokolov A, Aggarwal RR, Thomas G, True LD, Zhang L, Foye A, Alumkal JJ, Ryan CJ, Rettig M, Evans CP, Gleave ME, Baertsch R, Stuar J and Beer TM: Characterization of neuroendocrine prostate cancer (NEPC) in patients with metastatic castration resistant prostate cancer (mCRPC) resistant to abiraterone (Abi) or enzalutamide (Enz): Preliminary results from the SU2C/PCF/AACR West Coast Prostate Cancer. J Clin Oncol 33: 5003-5003, 2015.

6 Man YG, Fu SW, Liu AJ, Stojadinovic A, Izadjoo MJ, Chen L and Gardner WA: Aberrant expression of chromogranin A, miR$146 a$, and miR-146b-5p in prostate structures with focally disrupted basal cell layers: an early sign of invasion and hormone-refractory cancer? Cancer Genomics Proteomics 8: 235-244, 2011.

7 Wang HT, Yao YH, Li BG, Tang Y, Chang JW and Zhang J: Neuroendocrine Prostate Cancer (NEPC) progressing from conventional prostatic adenocarcinoma: factors associated with time to development of NEPC and survival from NEPC diagnosis-a systematic review and pooled analysis. J Clin Oncol 32: 3383-3390, 2014.

8 Horwich A, Parker C, de Reijke T and Kataja V: Prostate cancer: ESMO Clinical Practice Guidelines for diagnosis, treatment and follow-up. Ann Oncol 24(Suppl 6): vi106-114, 2013.

9 Heidenreich A, Bastian PJ, Bellmunt J, Bolla M, Joniau S, van der Kwast T, Mason M, Matveev V, Wiegel T, Zattoni F and
Mottet N: EAU Guidelines on Prostate Cancer. Part II: Treatment of Advanced, Relapsing, and Castration-Resistant Prostate Cancer. Eur Urol 65: 467-479, 2014.

10 Zhu M-L, Horbinski CM, Garzotto M, Qian DZ, Beer TM and Kyprianou N: Tubulin-targeting chemotherapy impairs androgen receptor activity in prostate cancer. Cancer Res 70: 7992-8002, 2010.

11 Darshan MS, Loftus MS, Thadani-Mulero M, Levy BP, Escuin D, Zhou XK, Gjyrezi A, Chanel-Vos C, Shen R, Tagawa ST, Bander NH, Nanus DM and Giannakakou P: Taxane-induced blockade to nuclear accumulation of the androgen receptor predicts clinical responses in metastatic prostate cancer. Cancer Res 71: 6019-6029, 2011.

12 Terry S, Maille P, Baaddi H, Kheuang L, Soyeux P, Nicolaiew N, Ceraline J, Firlej V, Beltran H, Allory Y, de la Taille A and Vacherot F: Cross modulation between the androgen receptor axis and protocadherin-PC in mediating neuroendocrine transdifferentiation and therapeutic resistance of prostate cancer. Neoplasia 15: 761-772, 2013.

13 Epstein JI, Amin MB, Beltran H, Lotan TL, Mosquera J-M, Reuter VE, Robinson BD, Troncoso P and Rubin MA: Proposed morphologic classification of prostate cancer with neuroendocrine differentiation. Am J Surg Pathol 38: 756-767, 2014.

14 Angelsen A, Syversen U, Haugen OA, Stridsberg M, Mjølnerød $\mathrm{OK}$ and Waldum HL: Neuroendocrine differentiation in carcinomas of the prostate: do neuroendocrine serum markers reflect immunohistochemical findings? Prostate 30: 1-6, 1997.

15 Berruti A, Mosca A, Porpiglia F, Bollito E, Tucci M, Vana F, Cracco C, Torta M, Russo L, Cappia S, Saini A, Angeli A, Papotti M, Scarpa RM and Dogliotti L: Chromogranin A Expression in Patients With Hormone Naïve Prostate Cancer Predicts the Development of Hormone Refractory Disease. J Urol 178: 838-843, 2007.

16 Scher HI, Morris MJ, Basch E and Heller G: End points and outcomes in castration-resistant prostate cancer: from clinical trials to clinical practice. J Clin Oncol 29: 3695-3704, 2011.

17 Martinez RLMC and Naranjo JD: A pretest for choosing between logrank and wilcoxon tests in the two-sample problem. METRON 68: 111-125, 2010.

18 Aparicio AM, Harzstark AL, Corn PG, Wen S, Araujo JC, Tu SM, Pagliaro LC, Kim J, Millikan RE, Ryan C, Tannir NM, Zurita AJ, Mathew P, Arap W, Troncoso P, Thall PF and Logothetis CJ: Platinum-based chemotherapy for variant castrate-resistant prostate cancer. Clin Cancer Res 19: 3621-3630, 2013.

19 Loriot Y, Massard C, Gross-Goupil M, Di Palma M, Escudier B, Bossi A and Fizazi K: Combining carboplatin and etoposide in docetaxel-pretreated patients with castration-resistant prostate cancer: a prospective study evaluating also neuroendocrine features. Ann Oncol 20: 703-708, 2009.

20 Ross RW, Beer TM, Jacobus S, Bubley GJ, Taplin M-E, Ryan CW, Huang J, Oh WK and Prostate Cancer Clinical Trials Consortium: A phase 2 study of carboplatin plus docetaxel in men with metastatic hormone-refractory prostate cancer who are refractory to docetaxel. Cancer 112: 521-526, 2008.

21 Kalkner KM, Acosta S, Thorsson O, Frederiksen H, Nilsson A, Gustavsson B, Elingsbo M, Stridsberg M and Abrahamsson PA: Octreotide scintigraphy and Chromogranin A do not predict clinical response in patients with octreotide acetate-treated hormone-refractory prostate cancer. Prostate Cancer Prostatic Dis 9: 92-98, 2006. 
22 Chou W-C, Chen J-S, Hung Y-S, Hsu J-T, Chen T-C, Sun C-F, Lu C-H and Hwang T-L: Plasma chromogranin A levels predict survival and tumor response in patients with advanced gastroenteropancreatic neuroendocrine tumors. Anticancer Res 34: 5661-5669, 2014.

23 Palmirotta R, Lovero D, Silvestris E, Felici C, Quaresmini D, Cafforio P and Silvestris F: Next-generation Sequencing (NGS) Analysis on Single Circulating Tumor Cells (CTCs) with No Need of Whole-genome Amplification (WGA). Cancer Genomics Proteomics 14: 173-179, 2017.

24 Gundem G, Van Loo P, Kremeyer B, Alexandrov LB, Tubio JMC, Papaemmanuil E, Brewer DS, Kallio HML, Högnäs G, Annala M, Kivinummi K, Goody V, Latimer C, O’Meara S, Dawson KJ, Isaacs W, Emmert-Buck MR, Nykter M, Foster C, Kote-Jarai Z, Easton D, Whitaker HC, Neal DE, Cooper CS, Eeles RA, Visakorpi T, Campbell PJ, McDermott U, Wedge DC, Bova GS and Bova GS: The evolutionary history of lethal metastatic prostate cancer. Nature 520: 353-357, 2015.

25 Schmid S, Omlin A, Blum D, Strasser F, Gillessen S and Rothermundt C: Assessment of anticancer-treatment outcome in patients with metastatic castration-resistant prostate cancer going beyond PSA and imaging, a systematic literature review. Ann Oncol 26: 2221-2247, 2015.

26 Burgio SL, Conteduca V, Menna C, Carretta E, Rossi L, Bianchi E, Kopf B, Fabbri F, Amadori D and De Giorgi U: Chromogranin A predicts outcome in prostate cancer patients treated with abiraterone. Endocr Relat Cancer 21: 487-493, 2014.
27 Heck MM, Thaler MA, Schmid SC, Seitz A-K, Tauber R, Kübler H, Maurer T, Thalgott M, Hatzichristodoulou G, Höppner M, Nawroth R, Luppa PB, Gschwend JE and Retz M: Chromogranin A and neurone-specific enolase serum levels as predictors of treatment outcome in patients with metastatic castration-resistant prostate cancer undergoing abiraterone therapy. BJU Int 119: 30-37, 2017.

28 Korse CM, Taal BG, Vincent A, van Velthuysen M-LF, Baas P, Buning-Kager JCGM, Linders TC and Bonfrer JMG: Choice of tumour markers in patients with neuroendocrine tumours is dependent on the histological grade. A marker study of Chromogranin A, Neuron specific enolase, Progastrin-releasing peptide and cytokeratin fragments. Eur J Cancer 48: 662-671, 2012.

29 Markwalder R and Reubi JC: Gastrin-releasing peptide receptors in the human prostate: relation to neoplastic transformation. Cancer Res 59: 1152-1159, 1999.

30 Giovacchini G, Giovannini E, Riondato $\mathrm{M}$ and Ciarmiello A: Radiopharmaceuticals for diagnosis and therapy of neuroendocrine differentiated prostate cancer. Curr Radiopharm 10: 6-15, 2016.

31 Yashi M, Muraishi O, Kobayashi Y, Tokue A and Nanjo H: Elevated serum progastrin-releasing peptide (31-98) in metastatic and androgen-independent prostate cancer patients. Prostate 51: 84-97, 2002.

Received June 19, 2017

Revised July 10, 2017

Accepted July 12, 2017 